\title{
Multiperiodicity in the newly discovered mid-late Be star V2104 Cygni ${ }^{\star}, \star \star$
}

\author{
K. Uytterhoeven ${ }^{1}$, E. Poretti ${ }^{1}$, E. Rodríguez ${ }^{2}$, P. De Cat $^{3}$, P. Mathias ${ }^{4}$, J. H. Telting ${ }^{5}$, V. Costa $^{2}$, and A. Miglio ${ }^{6}$ \\ 1 INAF-Osservatorio Astronomico di Brera, via E. Bianchi 46, 23807 Merate \\ e-mail: [katrien.uytterhoeven; ennio.poretti]@abrera.inaf.it \\ 2 Instituto de Astrofísica de Andalucía (CSIC), Apartado 3004, 18080 Granada, Spain \\ e-mail: [eloy;victor]@iaa.es \\ 3 Royal Observatory of Belgium, Ringlaan 3, 1180 Brussel, Belgium \\ e-mail: peter@oma.be \\ ${ }^{4}$ Observatoire de la Côte d'Azur, GEMINI, CNRS, Université Nice Sophia-Antipolis, BP 4229, 06304 Nice Cedex 4, France \\ e-mail: Philippe.Mathias@oca.eu \\ 5 Nordic Optical Telescope, Apartado 474, 38700 Santa Cruz de La Palma, Spain \\ e-mail: jht@not.iac.es \\ 6 Institut d'Astrophysique et de Géophysique de l'Université de Liège, Allée du 6 Août 17, 4000 Liège, Belgium \\ e-mail: a.miglio@ulg.ac.be
}

Received 17 April 2007 / Accepted 15 May 2007

\begin{abstract}
Aims. We obtained the first long, homogenous time-series of V2104 Cyg, consisting of 679 datapoints, with the $u v b y \beta$ photometers of the Sierra Nevada and San Pedro Mártir Observatories. Our aim was to detect and subsequently interpret the intrinsic frequencies of this previously unstudied variable star, which turned out to be a Be star. We evaluate its place among the variable B stars on the upper Main Sequence. To obtain additional information on physical parameters we collected a few spectra with the Elodie and FIES instruments.

Methods. We searched for frequencies in the $u v b y$ passbands using 2 different frequency analysis methods and used the $S / N>4$ criterion to select the significant periodicities. We obtained an estimate of the physical parameters of the underlying B star of spectral type between B5 and B7, by correcting for the presence of a circumstellar disk using a formalism based on the strength of the $\mathrm{H} \alpha$ line emission.

Results. We detected 3 independent frequencies with amplitudes below 0.01 mag, $v_{1}=4.7126 \mathrm{~d}^{-1}, v_{2}=2.2342 \mathrm{~d}^{-1}$ and $v_{3}=$ $4.671 \mathrm{~d}^{-1}$, and discovered that V2104 Cyg is a Be star. The fast rotation $\left(v \sin i=290 \pm 10 \mathrm{~km} \mathrm{~s}^{-1}\right.$, and $\left.27^{\circ}<i<45^{\circ}\right)$ hampered the investigation of the associated pulsational parameters $\ell$.

Conclusions. The most plausible explanation for the observed variability of this mid-late type Be star is a non-radial pulsation model.
\end{abstract}

Key words. stars: oscillations - stars: emission-line, Be - stars: individual: V2104 Cyg

\section{Introduction}

Several pulsators and variable stars of spectral type B occupy the region of the upper Main Sequence. Among them are the $\beta$ Cephei stars, the Slowly Pulsating B stars (SPBs) and the Be stars. The two former classes are well-established pulsators that show multiperiodic line-profile and light variations and whose pulsations are driven by the $\kappa$-mechanism acting on the iron bump. Most $\beta$ Cephei stars (spectral type B0.5-B3) pulsate in low-order $p$ - and $g$-modes with typical pulsation periods between 0.3 and 0.8 days. The largest fraction of this pulsational class is composed of moderate rotators $\left(v \sin i<150 \mathrm{~km} \mathrm{~s}^{-1}\right)$.

* Based on observations obtained at the Observatorio Astronómico Nacional San Pedro Mártir (Mexico), Observatorio de Sierra Nevada (Spain) and Observatoire de Haute Provence (France), and on observations made with the Nordic Optical Telescope, operated on the island of La Palma jointly by Denmark, Finland, Iceland, Norway, and Sweden, in the Spanish Observatorio del Roque de los Muchachos of the Instituto de Astrofísica de Canarias.

$\star \star$ Full Fig. 1 is only available in electronic form at http://www . aanda.org
The SPBs are less massive B stars (spectral type B2-B9) and slow rotators that pulsate in high radial order $g$-mode of low spherical harmonic degree $\ell$ with typical pulsation periods of 0.5 to 5 days. We refer to Stankov \& Handler (2005) and De Cat (2002) for overviews of the properties of the $\beta$ Cephei stars and $\mathrm{SPBs}$, respectively.

The Be stars are a more complex and less well understood class of near Main Sequence rapidly rotating B stars that produce a disk in their equatorial plane and hence show Balmer line emission. Be stars exhibit variations on different time-scales (hours-years), with a broad range of amplitudes. Explanations for this variability have been sought among the dynamics of the circumstellar disk, stellar wind, rotational modulation, magnetic field and/or pulsations (e.g. Porter \& Rivinius 2003). The highest fraction of Be stars is found in early-B type stars (B1-B2), while observed cases in the spectral type interval of B7-A2 are less frequent (Zorec 2000). However recently, a number of latetype Be stars were discovered during the ground-based preparatory work of the CoRoTsatellite (Neiner et al. 2005). The emission line B stars occupy the instability strips of $\beta$ Cephei and SPB pulsators and an obvious question arises whether or not the 
observed short-period Be variations resemble the properties of these well-established pulsators.

The short-periods observed in Be stars typically range from 0.2 to 5 days. Ground-based light curves mainly reveal monoperiodic signals, with periods close to the rotational period (the socalled $\lambda$ Eri variables, Balona 1990), and amplitudes of a few tens of mmag. These targets also show line-profile variations (LPV) of low spherical harmonic degree, similar to the LPV observed in $\beta$ Cephei variables, although the latter have shorter periods. The variability has been attributed to rotational modulation by Balona $(1990,1995)$ or non-radial pulsations (NRP) (Baade 1982). Spectroscopically, nearly all Be stars of early-B type show LPV (e.g. Rivinius et al. 2003). Contrary to groundbased photometric data, the LPV of several early-type Be stars reveal multiperiodic signals. The observed variability is interpreted in terms of NRP $g$-modes, typically with $\ell=m=+2$. Given the $g$-mode nature and the similar length of the pulsation periods, these $\mathrm{Be}$ stars appear to resemble the pulsational behaviour of SPBs. Until now, no clear cases of LPV in late-Be type stars (later than B6) have been found (Baade 1989; Rivinius et al. 2003). Recently, proof of the presence of low-amplitude additional periods and variability of late-type Be stars has been given by the MOST satellite as multiperiodic signals have been detected in the light of the Be stars HD163868 (B5Ve, Walker et al. 2005) and $\beta$ CMi (B8Ve, Saio et al. 2007). This result is very encouraging and gives high expectations for the outcome of the Be programme stars of the space mission CoRoT, which was launched on December 272006.

V2104 Cyg (HD190397, HIP98611, $\alpha_{2000}=20^{\mathrm{h}} 01^{\mathrm{m}} 45^{\mathrm{s}} .54$, $\left.\delta_{2000}=+57^{\circ} 39^{\prime} 06^{\prime \prime} 51, V=7.65 \mathrm{mag}\right)$ is listed in the HIPPARCoS catalogue (Perryman et al. 1997) as an unsolved variable of spectral type A0, probably a pulsating star, located at a distance of approximately $617 \pm 263 \mathrm{pc}$. According to Grenier et al. (1999), the spectrum of V2104 Cyg resembles a B8 Main Sequence star. Such a late-type B star or early-type A star would be a challenging object for many variable classes. To clarify this vague characterisation we obtained a photometric time-series and a few spectra. From the spectra it soon became evident that V2104 Cyg shows Balmer line emission (see Sect. 4). The study of V2104 Cyg is particularly interesting as (multiperiodic) variability is scarcely detected in late-type Be stars (see above). We present an analysis of the variable character of this newly discovered Be star and try to identify its place among the variable B stars on the upper Main Sequence.

\section{Photometric observations}

V2104 Cyg was observed in the framework of a double-site campaign in the autumn of 2005. Two twin Danish six-channel uvby $\beta$ photometers were used, one at the Sierra Nevada Observatory (SNO), Spain, and the other at the San Pedro Mártir Observatory (SPMO), Mexico, attached to the $90 \mathrm{~cm}$ and $1.5 \mathrm{~m}$ telescopes, respectively. Both photometers are equipped for simultaneous measurements in uvby or the narrow and wide $\mathrm{H} \beta$ channels (Nielsen 1983). Nevertheless, most of the data were collected in the four uvby filters in order to investigate the variability behaviour of this star. Only a few points were obtained in $\mathrm{H} \beta$ at SNO for calibration purposes of the photometric indices.

HR $7634(V=6.16 \mathrm{mag}, \mathrm{A} 4 \mathrm{Vn})$ and $\mathrm{HR} 7692(\mathrm{~V}=$ 6.19 mag, F4V) were used as comparison stars in both sites. A total of 414 uvby datapoints of V2104 Cyg were collected on 8 nights at SNO, from October 3 to 26, and 265 uvby datapoints on 7 nights at SPMO, from November 4 to 14. In total, about
$54 \mathrm{~h}$ of useful data were acquired. The light curves are given in Fig. 1.

The quality of the nights was excellent at SPMO. Bouguer's lines, i.e. the least-squares fit to the observations of a same star versus airmass for each night, yielded correlation coefficients in the interval $0.994-1.000$, whereby 23 times out of 28 in the $0.998-1.000$ interval. Mean extinction coefficients were $0.472 \mathrm{mag}$ in $u$ light, $0.267 \mathrm{mag}$ in $v$ light, $0.165 \mathrm{mag}$ in $b$ light and $0.116 \mathrm{mag}$ in $y$ light. Similar mean coefficients, with standard deviation given between brackets, were obtained at SNO: $0.484 \mathrm{mag}(0.013 \mathrm{mag}), 0.278 \mathrm{mag}(0.009 \mathrm{mag})$, $0.171 \mathrm{mag}(0.007 \mathrm{mag})$ and $0.121 \mathrm{mag}(0.010 \mathrm{mag})$ for filters $u, v, b$ and $y$, respectively, with the airmass ranging from about 1.05 to 1.5 each observing night.

An analysis of the magnitude differences between the two comparison stars was carried out to get an additional feeling of the quality of the dataset. The overall standard deviations of the 268 magnitude differences obtained at SPMO are: $6.7 \mathrm{mmag}$ in $u$ light, $2.0 \mathrm{mmag}$ in $v$ light, $1.9 \mathrm{mmag}$ in $b$ light and $1.8 \mathrm{mmag}$ in $y$ light. The high scatter in the $u$ filter is due to some leakage problems in the photomultiplier which affected the measurements in a random way. The standard deviations of each night lie between 4.6-7.5, 1.4-2.3, 1.4-2.2 and 1.2-2.1 mmag in $u, v, b$ and $y$ filters, respectively.

For the SNO data, we measured the two comparison stars 427 times and the magnitude differences yielded standard deviations of $6.3,2.3,2.3$ and 2.9 mmag in $u, v, b$ and $y$ filters, respectively. Excluding the night JD 24453652 (where values are 1.5-2.0 times greater), the standard deviations of each night lie between 5.5-6.7, 1.7-2.6, 1.4-2.3 and 2.1-2.7 mmag in $u, v$, $b$ and $y$ filters, respectively.

Since small differences are present in the mean values measured at SNO an SPMO, the magnitude differences between the comparison stars have been aligned. The combined timeseries have standard deviations of 6.4, 2.2, 2.1 and 2.3 mmag in uvby, respectively. Therefore, our photometric timeseries are characterised by very good precision, close to the best value we can obtain from the ground. The corresponding periodogram is shown in the bottom panel of Fig. 2.

\section{Frequency analysis}

The frequency search was carried out using Lomb-SCARGLE (Scargle 1982) as well as the least-squares power spectrum method (Vaníček 1971). The latter method allows the detection of the constituents of the light curve one by one, whereby only the values of the detected frequencies are introduced as known constituents in each new search. This procedure differs from the SCARGLE method as it does not require any data prewhitening as amplitudes and phases of the known constituents are recalculated for each new trial frequency, whereby the exact amount of signal for any detected term is always subtracted.

We first double-checked the reliability of the comparison stars. The frequency analysis of the combined timeseries between the two comparison stars did not reveal any significant term. White noise is around $0.3 \mathrm{mmag}$ in the $v b y$ data and amplitudes of the highest peaks are in the $0.6-0.8 \mathrm{mmag}$ interval. The $u$ data show a more noisy behaviour. The highest peaks occur at different frequencies in the different filters, suggesting a random enhancement. As an illustration, we show the frequency spectrum of the $v$ data in the bottom panel of Fig. 2. Thus, we can state that the two comparison stars are constant at the level of a few 0.1 mmag. 


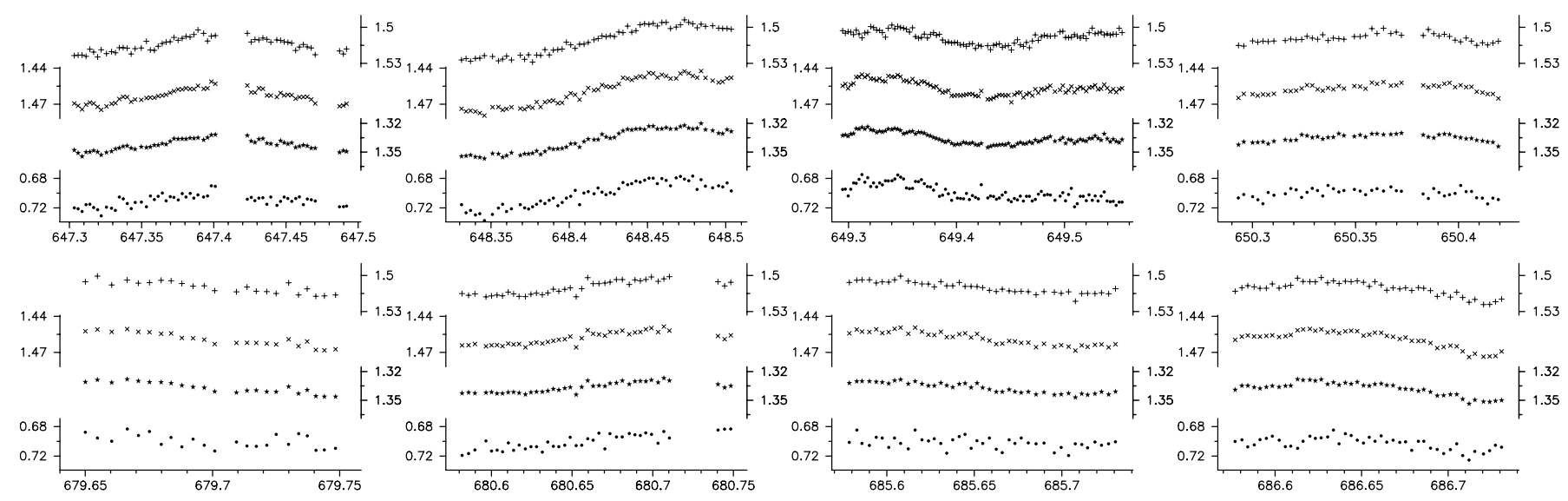

Fig. 1. Light curves of V2104 Cyg obtained at SNO and SPMO in October-November 2005. The $u$ (dots), $v$ (stars), $b$ (crosses) and $y$ (+) magnitude differences are plotted in time (HJD-2453000). Note the difference in scale between the $u$ and $v b y$ data: tickmarks in the $u$ filter and $v b y$ filters correspond to $0.04 \mathrm{mag}$ and $0.03 \mathrm{mag}$ respectively. The light curves of all 15 nights are only available in the on-line version of the paper.

The frequency analysis of the V2104 Cyg timeseries turned out to be more complex. We initially analysed the SNO and SPMO datasets separately. We searched for frequencies in the interval $0.01-25.0 \mathrm{~d}^{-1}$ with a frequency step of $0.01 \mathrm{~d}^{-1}$. We detected similar structures in the least-squares power spectra and the SCARGLE periodograms of the datasets in the different filters and found evidence for two frequencies centered at $v_{1}=$ $4.71 \mathrm{~d}^{-1}$ and $v_{2}=2.23 \mathrm{~d}^{-1}$, or their one-day aliases. Additional frequency peaks were visible but were below the significance threshold (see below).

Subsequently, a preliminary solution for each dataset was obtained by means of these two frequencies only and the two datasets were re-aligned at the same mean brightness level. In particular, we obtained the same mean magnitude for the $y$ data, within error bars, while small misalignements were measured in the other data.

In a second step we analysed the aligned full dataset, consisting of 679 datapoints and spanning 42 days. Given the improved frequency resolution we adopted a frequency step of $0.001 \mathrm{~d}^{-1}$. The spectral window of our timeseries is shown in the upper panel of Fig. 2. Besides $v_{1}=4.713 \mathrm{~d}^{-1}$ and $v_{2}=2.234 \mathrm{~d}^{-1}$, with amplitudes of 7.6 and $5.7 \mathrm{mmag}$ in the $v$ filter respectively, we also found in all filters and using both frequency methods, a third term $v_{3}=4.670 \mathrm{~d}^{-1}$, with an amplitude of $2.4 \mathrm{mmag}$ in the $v$ time series. The amplitudes of all 3 frequencies satisfy the $S / N>3.6$ significance criterion and also the more severe $S / N>4$ criterion (Breger et al. 1993; Kuschnig et al. 1997; De Cat \& Cuypers 2003 ) in the $v b y$ filters. However, the low-amplitude of $v_{3}$ does not pass $S / N>4$ in the noisy $u$ filter (see last row Table 1). The $S / N$-level was computed as the average amplitude over a frequency interval with a width of $5 \mathrm{~d}^{-1}$ in an oversampled SCARGLE periodogram obtained after final prewhitening. We note that the $S / N$-level is a factor 1.5 higher than the white noise detected in the comparison stars (bottom panel Fig. 2). Comparing the white noise with the residual time-series of V2104 Cyg (last but one bottom panel Fig. 2), there might be even more frequencies present in the signal of V2104 Cyg, with amplitudes currently below the significance threshold.

For the moment we accept a model with $v_{1}, v_{2}$ and $v_{3}$. We did not find a direct relation between the three frequencies, which can be considered independent of each other. To optimise this triperiodic model we used a code, kindly made available by Dr. Jan Cuypers (Royal Observatory of Belgium), that searches simultaneously a set of frequencies around the input values to find the multiperiodic combination that fits the data best. The optimised solution is: $v_{1}=4.7126 \pm 0.0004 \mathrm{~d}^{-1}$, $v_{2}=2.2342 \pm 0.0005 \mathrm{~d}^{-1}$ and $v_{3}=4.671 \pm 0.001 \mathrm{~d}^{-1}$. A similar fit using a different code (MTRAP, Carpino et al. 1987) yielded coincident results. The given accuracy of the frequencies is the average of the estimated accuracies in the $u v b y$ filters. The frequency accuracy in a particular filter $x$ is calculated as $\sigma_{v, x}=$ $\left.\sqrt{(6)} \sigma_{\text {std }, x} / \pi \sqrt{(} N\right) A_{v, x} \Delta T$ (Montgomery \& O'Donoghue 1999), with $\sigma_{\text {std }, x}$ the standard deviation of the final residuals, $A_{v, x}$ the amplitude of the frequency $v$ in filter $x$ and $\Delta T$ the total timespan of the observations. The solution of the least-squares fit of this triperiodic model is given in Table 1 . The phases $\phi_{i}$ are calculated according to the formula $A_{0}+\sum_{i=1,3} A_{i}\left(\cos 2 \pi\left(t-T_{0}\right) v_{i}+\right.$ $\left.\phi_{i}\right)$. Note that the phase values for $v_{3}$ in the four passbands are in excellent agreement, supporting the reality of this small amplitude term. The triperiodic fit applied to the individual datasets of SNO and SPMO yielded the same mean brightness levels in all filters, i.e. the alignment procedure was insensitive to $v_{3}$. The SCARGLE periodogram of the $v$ filter at different stages of the frequency search and the phase diagrams with the 3 frequencies are given in Fig. 2. For reasons of visibility of the peaks around $v_{3}$ we only show the $(S / N>3.6)$-level, indicated by the light grey horizontal line.

We also analysed the HIPPARCos data in search for traces of intrinsic variability. The resulting SCARGLE periodogram resembled very much the window function and no frequencies could be detected. The poor spectral window and the poorer accuracy of the HIPPARCOS data did not allow detection of any of our three small amplitude terms.

\section{Elodie and FIES spectra: evidence for a Be star}

In addition to the photometric time-series we obtained 3 spectra during 3 consecutive nights in June 2006 with the Elodie spectrograph $(R \sim 45000)$ at the $193 \mathrm{~cm}$ telescope of the Observatoire de Haute Provence (OHP), France, and one spectrum in November 2006 with the FIES spectrograph in medium resolution mode $(R \sim 45000)$ at the Nordic Optical Telescope located at the Observatorio del Roque de los Muchachos, La Palma, Spain. We combined the 3 Elodie spectra, after checking that there are no clear differences visible, resulting in an average spectrum with a signal-to-noise ratio of 70 . The signal-to-noise ratio of the FIES spectrum is about 150 . 


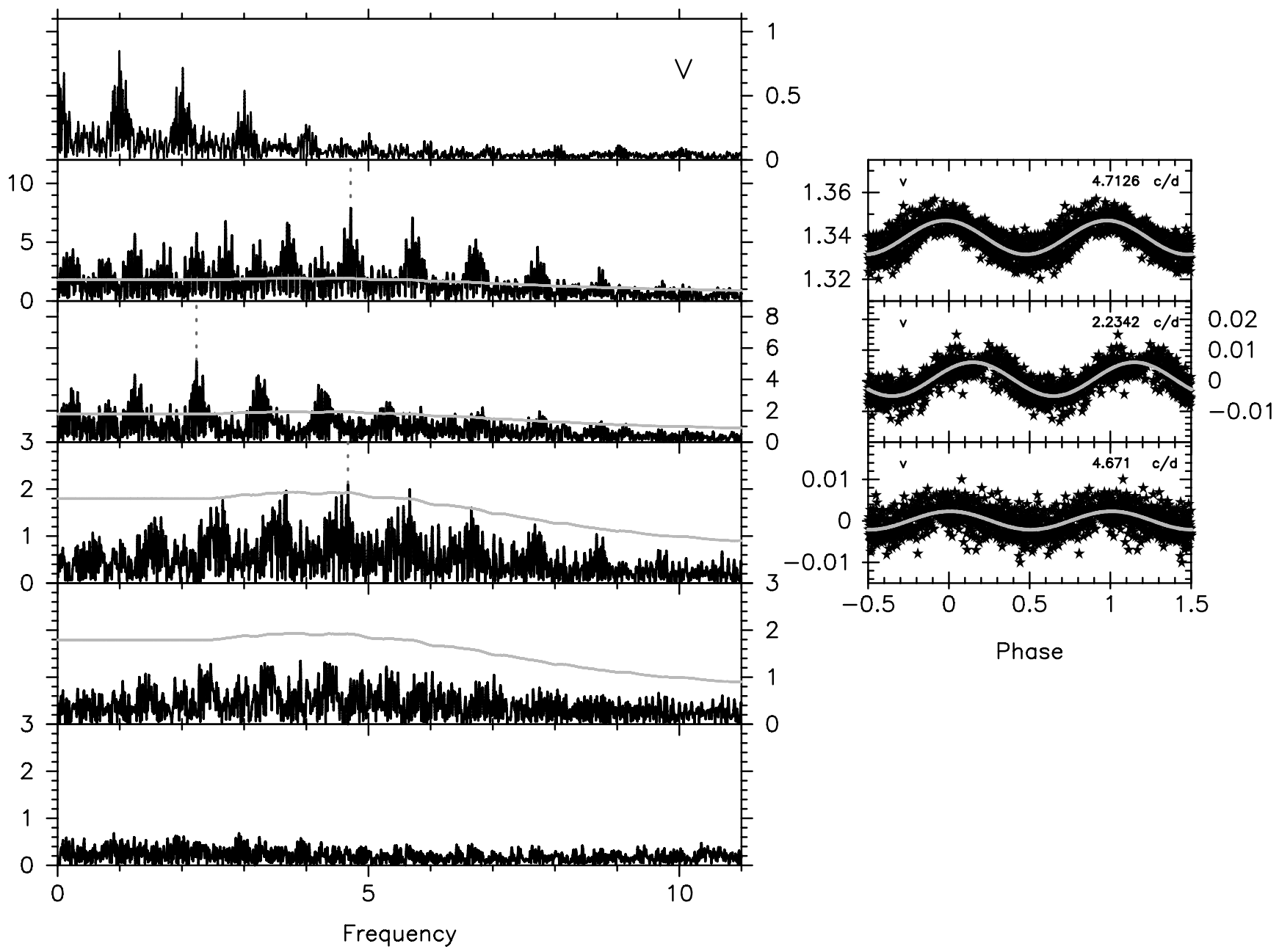

Fig. 2. Left panel: Window function (top) and SCARGLE periodograms of V2104 Cyg at different stages of the frequency search in the Strömgren $v$ filter. The amplitudes are expressed in mmag. The detected frequencies $v_{1}=4.7126 \pm 0.0004 \mathrm{~d}^{-1}, v_{2}=2.2342 \pm 0.0005 \mathrm{~d}^{-1}$ and $v_{3}=4.671 \pm$ $0.001 \mathrm{~d}^{-1}$ are indicated by dashed vertical lines. The last but one bottom periodogram shows the final residuals after prewhitening with $v_{1}, v_{2}$ and $v_{3}$. The light grey horizontal lines represent the $(S / N>3.6)$-level. In the bottom periodogram we present the variability of the comparison stars, illustrating that they are constant at the level of a few $0.1 \mathrm{mmag}$. Right panel: Phase diagrams of the 3 detected frequencies in the $v$ filter. Black stars correspond to observations and the grey full lines represent the least-squares fit. The amplitudes are expressed in mag.

The $\mathrm{H} \alpha$ and $\mathrm{H} \beta$ line profiles clearly show a double-peaked emission line (see Fig. 3). Hence, we identify V2104 Cyg as a Be star. The emission effect appears to be much stronger in $\mathrm{H} \alpha$ than in $\mathrm{H} \beta$. In the FIES spectra we also see a suggestion of emission in the absorption profile of $\mathrm{H} \gamma$ but this needs to be confirmed. A comparison between the $\mathrm{H} \alpha$ profile (upper panel Fig. 3) - obtained in June 2006 (Elodie spectra, grey) and in November 2006 (FIES spectrum, black) - shows a radial velocity shift and small variations in the shape of the emission peaks. A dedicated spectroscopical campaign is necessary to study the dynamical character of the circumstellar disk.

As the line profiles are strongly rotationally broadened, only the Balmer lines, He I lines and the absorption line of Mg II 4481 $\AA$ are prominently present and not lost in the noise of the spectra. To derive an estimate of $v \sin i$ we calculated theoretical line profiles for a rotating star by means of the code described by Schrijvers \& Telting (1999), whereby we assumed a neglegible contribution to the line shape from NRP. We used limbdarkening coefficient $\alpha=0.444$, taken from Díaz-Cordovés et al. (1995) for the physical parameters given in Table 2. As Collins et al. (1991) have suggested the He I $4027 \AA$, He I $4471 \AA$ and Mg II $4481 \AA$ profiles as most suitable candidates for the determination of $v \sin i$, we focussed on these line profiles only. From the best fits of the observed profiles we estimated $v \sin i=$ $290 \mathrm{~km} \mathrm{~s}^{-1}$, with formal errors of $10 \mathrm{~km} \mathrm{~s}^{-1}$.

We calculated the $E W$ of the $\mathrm{H} \alpha$ profile and obtained $E W \sim$ $-6.0 \pm 0.5 \AA$. The red peak of the emission is higher than the violet peak with peak intensities of $R=2.42$ and $V=2.36$. The peak separation $\Delta V$ between the red and violet peak is about $105 \mathrm{~km} \mathrm{~s}^{-1}$. As $\Delta V$ is a measure of the radius of the circumstellar disk, namely $(2 v \sin i / \Delta V)^{2}=\left(R_{\text {disk }} / R_{\star}\right)$ assuming a Keplerian rotation (e.g. Zamarov et al. 2001), we estimate the radius of the circumstellar disk to be about 34 times the stellar radius.

Given the double-peaked nature of the emission line, with small side-lobes on each side (Fig. 3), we expect to see the circumstellar disk under an inclination angle $i$ between $45^{\circ}$ and $\sim 20^{\circ}$ (e.g. Hanuschik et al. 1996).

We compared the FIES spectrum of V2104 Cyg with a set of spectra from the noao spectral library (Valdes et al. 2004) and deduced a spectral type between B5 and B7. Hence we obtained a hotter star than previously reported (A0, Perryman et al. 1997; B8, Grenier et al. 1999). The misclassification of a late-B type star as an early-A type star is not uncommon among fast rotators. Being a mid-late Be star, V2104 Cyg remains an interesting 
Table 1. Amplitudes (in mag) and phases (in rad) of the frequencies $v_{1}=4.7126 \mathrm{~d}^{-1}, v_{2}=2.2342 \mathrm{~d}^{-1}$ and $v_{3}=4.671 \mathrm{~d}^{-1}$ calculated from the $u v b y$ time-series of V2104 Cyg by means of a triperiodic least-squares fit. For each filter the mean magnitude difference is given together with the rms, of the residuals. The last row indicates the calculated $(S / N=4)$-level at $v_{3}=4.671 \mathrm{~d}^{-1}$. Phase $=0$ corresponds to $T_{0}=2453647.3034$.

\begin{tabular}{|c|c|c|c|c|c|c|c|c|c|}
\hline \multirow[b]{2}{*}{ Term } & \multirow[b]{2}{*}{$\begin{array}{l}\text { Freq. } \\
{\left[\mathrm{d}^{-1}\right]}\end{array}$} & \multicolumn{2}{|c|}{$u$ light } & \multicolumn{2}{|c|}{$\mathrm{v}$ light } & \multicolumn{2}{|c|}{$b$ light } & \multicolumn{2}{|c|}{$y$ light } \\
\hline & & $\begin{array}{l}\text { Ampl. } \\
{[\mathrm{mag}]}\end{array}$ & $\begin{array}{l}\text { Phase } \\
\text { [rad] }\end{array}$ & $\begin{array}{l}\text { Ampl. } \\
{[\mathrm{mag}]}\end{array}$ & $\begin{array}{l}\text { Phase } \\
\text { [rad] }\end{array}$ & $\begin{array}{l}\text { Ampl. } \\
{[\mathrm{mag}]}\end{array}$ & $\begin{array}{l}\text { Phase } \\
\text { [rad] }\end{array}$ & $\begin{array}{l}\text { Ampl. } \\
{[\mathrm{mag}]}\end{array}$ & $\begin{array}{l}\text { Phase } \\
\text { [rad] }\end{array}$ \\
\hline \multirow[t]{2}{*}{$v_{1}$} & 4.7126 & 0.0086 & 0.17 & 0.0073 & 0.26 & 0.0071 & 0.25 & 0.0071 & 0.27 \\
\hline & \pm .0004 & \pm .0004 & \pm .09 & \pm .0002 & \pm .04 & \pm .0002 & \pm .04 & \pm .0002 & \pm .05 \\
\hline \multirow[t]{2}{*}{$v_{2}$} & 2.2342 & 0.0098 & 5.41 & 0.0059 & 5.31 & 0.0058 & 5.28 & 0.0051 & 5.28 \\
\hline & \pm .0005 & \pm .0005 & \pm .05 & \pm .0002 & \pm .03 & \pm .0002 & \pm .04 & \pm .0002 & \pm .04 \\
\hline \multirow{2}{*}{$v_{3}$} & 4.671 & 0.0026 & 6.2 & 0.0024 & 6.1 & 0.0024 & 6.1 & 0.0024 & 6.2 \\
\hline & \pm .001 & \pm .0004 & \pm .3 & \pm .0002 & \pm .1 & \pm .0002 & \pm .1 & \pm .0002 & \pm .2 \\
\hline \multirow{2}{*}{\multicolumn{2}{|c|}{$\begin{array}{c}\text { Mean } \Delta \mathrm{m}\left(A_{0}\right) \\
\text { Residual rms [mag] }\end{array}$}} & \multirow{2}{*}{\multicolumn{2}{|c|}{$\begin{array}{c}0.7054 \pm .0003 \\
0.0065\end{array}$}} & \multirow{2}{*}{\multicolumn{2}{|c|}{$\begin{array}{c}1.3398 \pm .0001 \\
0.0025\end{array}$}} & \multirow{2}{*}{\multicolumn{2}{|c|}{$\begin{array}{c}1.4610 \pm .0001 \\
0.0026\end{array}$}} & \multirow{2}{*}{\multicolumn{2}{|c|}{$\begin{array}{c}1.5124 \pm .0001 \\
0.0028\end{array}$}} \\
\hline & & & & & & & & & \\
\hline \multicolumn{2}{|c|}{$S / N=4 @ v_{3}$} & \multicolumn{2}{|c|}{0.0038} & \multicolumn{2}{|c|}{0.0022} & \multicolumn{2}{|c|}{0.0022} & \multicolumn{2}{|c|}{0.0019} \\
\hline
\end{tabular}
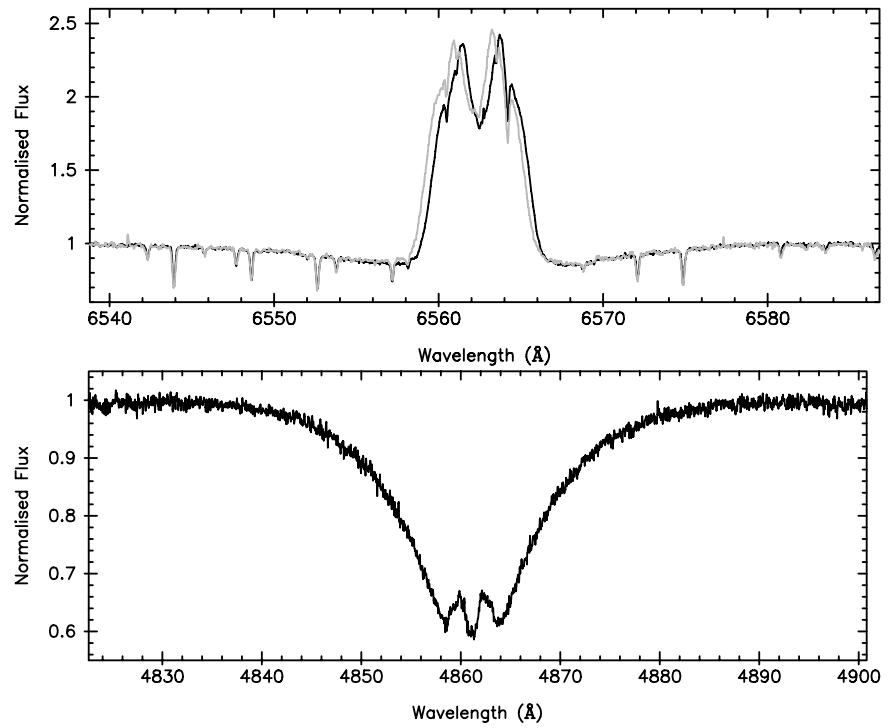

Fig. 3. The $\mathrm{H} \alpha$ and $\mathrm{H} \beta$ profiles of V2104 Cyg clearly show emission. The black profiles are FIES profiles while the grey $\mathrm{H} \alpha$ profile is taken with the Elodie spectrograph. Between June 2006 and November 2006 the emission peak shifted and changed slightly in shape, indications for the dynamical character of the circumstellar disk.

target given that cooler Be stars are less frequently observed than early-type Be stars.

\section{Physical parameters}

First, we calculated $T_{\text {eff }}, \log g$ and $M_{V}$ of V2104Cyg from the Strömgren indices, whereby using HR 7634 and HR 7692 as calibration stars. The observed Strömgren indices (all expressed in mag throughout the text) are $(b-y)=0.000, m_{1}=0.096$ and $c_{1}=0.534$. They have been calculated from the mean magnitude differences reported in Table 1 and from $u v b y$ standard photometry of HR 7634. A $\beta=2.681$ value simulaneous to uvby photometry was determined from dedicated observations at SNO. We obtained dereddened values starting from the above indices and applying the TEMPLOGG method (Kupka \& Bruntt 2001): $(b-y)_{0}=-0.057, m_{0}=0.115, c_{0}=0.523$. The interstellar reddening is non-negligible, $E_{b-y}=+0.057 \mathrm{mag}$, since the star lies only $14^{\circ}$ above the galactic plane. Additionally, the TEMPLOGG method allowed us to derive $M_{V}=-1.44 \mathrm{mag}$, $\log g=3.37$ and $T_{\text {eff }}=14000 \mathrm{~K}$. According to these values,

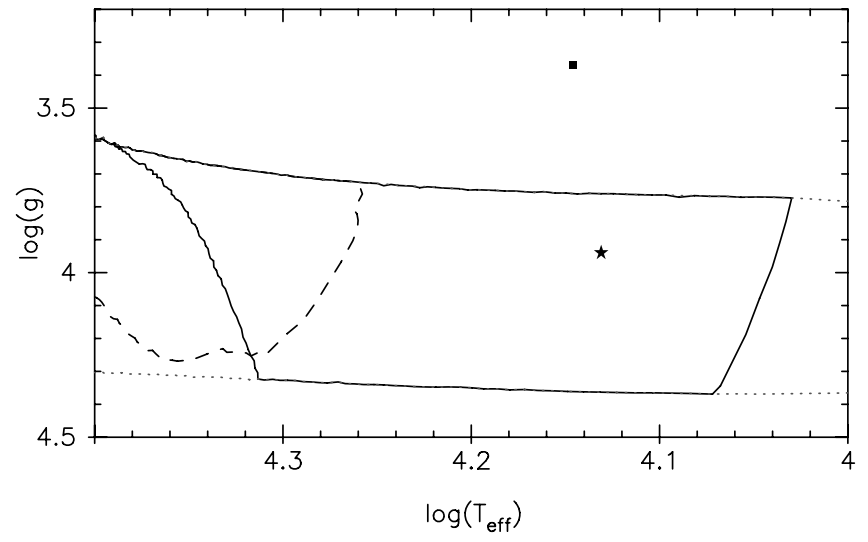

Fig. 4. Possible position of V2104 Cyg in the Hertzsprung-Russell diagram. The black square indicates the position derived from the observed photometric indices while the star indicates the position after correction for the contribution of the stellar disk. The full black lines and the dashed black lines represent the instability strips for the SPB and $\beta$ Cephei modes respectively, with a frequency between 0.25 and $25 \mathrm{~d}^{-1}$ and $\ell \leq 4$ computed for Main Sequence models (De Cat et al. 2007). The dark grey dotted lines show the ZAMS and the TAMS.

V2104 Cyg is located far above the instability strip of the SPBs in the Hertzsprung-Russell diagram (black square in Fig. 4).

As we are dealing with a Be star, we expect that the presence of the circumstellar envelope affects the observed photometric indices. This might also explain why SIMBAD reports scattered values $(2.610,2.648$ and 2.705$)$ of the $\beta$ index for our target. To deduce the indices from the underlying B star we calculated the transformation formulae given by Fabregat \& Reglero (1990), which are based on the assumption that the contribution of the circumstellar disk is proportional to the $E W$ of the $\mathrm{H} \alpha$ emission peak. Assuming an $E W$ of $\mathrm{H} \alpha$ of $-6.0 \pm 0.5 \AA$ we obtained the following new values of the Strömgren indices: $(b-y)_{\star}=-0.01 \pm 0.01,\left(m_{1}\right)_{\star}=0.10 \pm 0.05,\left(c_{1}\right)_{\star}=0.57 \pm 0.05$ and $\beta_{\star}=2.73 \pm 0.01$. Applying the TEMPLOGG method on these values resulted in dereddened values $(b-y)_{0, \star}=-0.05 \pm 0.01$, $\left(m_{0}\right)_{\star}=0.12 \pm 0.05$ and $\left(c_{0}\right)_{\star}=0.564$ in addition to $\left(M_{V}\right)_{\star}=$ $-0.49 \mathrm{mag},(\log g)_{\star}=3.94 \pm 0.05$ and $\left(T_{\text {eff }}\right)_{\star} \sim 13500 \mathrm{~K}$. After correction for the contribution of the circumstellar disk, the position of the underlying B star falls nicely inside the SPB instability strip ( $\star$ in Fig. 4).

From the corrected value $M_{V, \star}=-0.49$ mag we can estimate the luminosity and the mass of V2104 Cyg using the relation for 
Table 2. Estimates of the physical parameters of the Be star V2104 Cyg, after correction for the contribution of the circumstellar disk. The effective temperature is given in $\mathrm{K}$; the mass and radius are expressed in solar units; $v \sin i$ is given in $\mathrm{km} \mathrm{s}^{-1}$.

\begin{tabular}{cccccc}
\hline \hline$T_{\text {eff }}$ & $\log g$ & Mass & Radius & $L / L_{\odot}$ & $v \sin i$ \\
\hline $13500 \pm 1350$ & $3.94 \pm 0.39$ & $\sim 4$ & $2.5 \pm 0.5$ & $\sim 127$ & $290 \pm 10$ \\
\hline
\end{tabular}

Main Sequence stars between absolute magnitude and luminosity and the mass-luminosity relation. Assuming that V2104 Cyg is a MS star we find: $L / L_{\odot} \sim 127$ and $M / M_{\odot} \sim 4$. Using the formula $\log \left(R / R_{\odot}\right)=\frac{1}{2} \log \left(L / L_{\odot}\right)-2 \log \left(T_{\text {eff }} / T_{\text {eff } \odot}\right)$, we estimate $R / R_{\odot} \sim 2.1$. The value of the mass is compatible with the expected values for a B5-B7 star according to the tabulation of Harmanec (1988), while we obtained a slightly lower radius (2.1 versus $\left.3.0 R_{\odot}\right)$. From these estimates of mass and radius, the expected upper limit of the inclination $i<45^{\circ}$ (see above), and with $v \sin i=290 \pm 10 \mathrm{~km} \mathrm{~s}^{-1}$, we can derive that V2104 Cyg rotates at least at $65 \%$ of its critical velocity, using $v_{\text {crit }}=\sqrt{G M_{\star} / R_{\star}}$. This fact confirms the tight connection between fast rotation and the Be phenomenon. Moreover, an interpretation in terms of multiperiodic pulsations would confirm and strengthen the relation between pulsation and mass loss, and demonstrate that this relation also exists in mid-late Be stars. Subsequently, we obtain from the derived $v \sin i$ interval and imposing $v<v_{\text {crit }}$ a lower limit for the inclination angle: $i>27^{\circ}$.

Next, from $v \sin i=290 \pm 10 \mathrm{~km} \mathrm{~s}^{-1}$, a stellar radius in the interval $[2,3] R_{\odot}$, and an inclination angle between $27^{\circ}$ and $45^{\circ}$ we arrive at a (very) rough estimate of the rotational frequency: $\Omega \in[2.6,6.5] \mathrm{d}^{-1}$. We note that the observed intrinsic frequencies $v_{1}$ and $v_{3}$ lie in this interval. It is not uncommon for Be stars that the rotational period is dominantly present in the light variations (e.g. Balona 1990). This possibility is further discussed in Sect. 6.

We stress that the derived physical parameters tabulated in Table 2 are only a first estimate as the contribution and properties of the circumstellar disk are still unknown and as the properties derived from the low $S / N$ noise spectra have to be taken with caution.

\section{Discussion and conclusions}

In the light curves of V2104 Cyg, we detected 3 intrinsic frequencies with amplitudes below 0.01 mag: $v_{1}=4.7126 \mathrm{~d}^{-1}$, $v_{2}=2.2342 \mathrm{~d}^{-1}$ and $v_{3}=4.671 \mathrm{~d}^{-1}$. We also discovered that this object is an intrinsically fast rotating Be star. V2104Cyg is a particularly interesting case as there are not many precedents for detection of multiperiodicity in mid-late type Be stars based on ground-based photometric observations. Indeed, from previous efforts (Cuypers et al. 1989; Balona et al. 1992) it appears that detection of multi-periodic signals from ground-based photometry in Be stars is not easy. Only recently, GutiérrezSoto et al. (2006) claimed to have detected unambiguously several NRP periods in the light curves of NW Ser (spectral type B3) and V1446 Aql (spectral type B5). If V2104 Cyg is a multiperiodic non-radial pulsator as well, it would be the third known Be pulsator with multiperiodic signals detected in its groundbased light curves.

How can we explain the variability of V2104 Cyg? Short periods of $0.2^{d}$, like $v_{1}$ and $v_{3}$ are typically detected in early-B type stars such as $\beta$ Cephei pulsators or early-Be stars, but are quite unusual in late-type B(e) stars. As V2104 Cyg lies in the instability domain of the SPBs, far from the overlap domain with the $\beta$ Cephei pulsators, we expect to detect longer periods, such as $v_{2}$. Rivinius et al. (2003) identified $\ell=m=2 g$-modes associated to periods longer than $0.4^{d}$ in a selected set of Be stars. Given that V2104 Cyg has a spectral type between B5 and B7 it is worth noting that the NRP stars in Rivinius et al. (2003) have a spectral type earlier than B6.

According to Saio et al. (2007) it is likely that all rapidly rotating Be stars show NRP. For fast rotating stars, the comparison of the observed frequencies $v_{\mathrm{obs}}$ with the eigenfrequencies of the star $v_{\text {eigen }}$ provided by theoretical calculations is not straightforward (e.g. Berthomieu 1978; Dintrans \& Rieutord 2000). The rotation lifts the degeneracy of the eigenfrequencies of the modes in $m$ and gives rise to a perturbation of the frequencies (e.g. Saio 1981; Dziembowski \& Goode 1992; Soufi et al. 1998). These frequency shifts depend on $m$, the rotation frequency $\Omega$, and other parameters, and can be very large, especially for $g$-modes. This perturbation can explain why the observed frequencies $v_{1}$ and $v_{3}$ of V2104 Cyg are larger than expected for stars within the instability strip of SPBs. Moreover, when the oscillation frequency is of the same order as the rotational frequency, the whole perturbative approach is invalid and the familiar structure of modes $(n, \ell)$ disappears (Dintrans $\&$ Rieutord 2000). Hence, rotation has a significant effect on the non-adiabatic analysis and mode identification. Consequently it is not easy to assign $l$-values to the observed frequencies of a fast-rotating star, like V2104 Cyg. In particular, the fast rotation of V2104 Cyg prevents the application of a mode-identification method that does not account for higher order rotational effects, such as the method of photometric amplitude ratios made available by Dupret et al. (2003). A discussion of the implications of applying standard mode-identification techniques, used to identify modes in non-rotating stars, to fast-rotating stars can be found in Townsend (2003).

It is interesting to point out that for V2104 Cyg the amplitudes associated with $v_{3}$ have, unlike those associated with $v_{1}$ and $v_{2}$, a similar value in all four filters (see Table 1). In general, the amplitudes of NRP modes in B stars decrease from the $u$ filter towards the $v b y$ filters. However, similar pulsation amplitudes at different wavelengths are predicted for some modes of theoretical models. Several examples can be found in De Cat et al. (2007; e.g. HD 14053 and HD 89688).

An alternative explanation of the observed variability of V2104 Cyg might be in terms of a model based on rotational modulation. In many Be stars the detected period is consistent with the expected period of rotation of the star (Cuypers et al. 1989; Balona et al. 1992). As seen in Sect. 5, $v_{1}$ or $v_{3}$ might be related with $\Omega$. Our dataset did not allow a sufficiently tight constraint on $\Omega$ to confirm this. The frequency $v_{2}$, however, is much lower than the expected rotational frequency. Therefore, a model based on rotational modulation could not explain the light variability of V2104 Cyg.

The unambiguous detection of photospheric multiperiodicity in a Be star is in favour of a NRP interpretation (e.g. Porter \& Rivinius 2003). As we detected 3 independent frequencies in the light curves of V2104 Cyg, the NRP hypothesis currently seems the most plausible explanation. The interpretation of pulsational variability in Be stars is complicated by fast rotation. Recently, theoretical progress on this topic has been made by Townsend (2005), who proposed a new explanation for unstable modes in mid- to late B-type SPB and Be stars in terms of retrograde mixed modes instead of $g$-modes and by Dziembowski et al. (2007), who found theoretical backing for the observed frequencies in the MOST data of the Be star HD163868 (Walker et al. 2005). The case study of V2104 Cyg, 
a fast-rotating mid-late Be star with 3 excited modes, is another challenge for theoreticians in the game of matching theory with observations.

A high-resolution spectroscopic time-series of V2104 Cyg could be a future possible observational progress. As LPV are rarely detected in late-type Be stars (Baade 1989; Rivinius et al. 2003) it would be an interesting excercise to see if V2104 Cyg is an exceptional case in this respect and indeed shows LPV. If present, a line-profile analysis and subsequent mode-identification will allow $m$-values to be assigned to the detected modes. Additionally, a study of the behaviour of the $\mathrm{H} \alpha$ emission peak will allow the properties and behaviour of the circumstellar disk to be studied.

Acknowledgements. We thank the referee for providing constructive comments which helped improve our conclusions. We thank Marc-Antoine Dupret and Josefina Montalban for sharing their opinion on the mode-identification. K.U. acknowledges financial support from a European Community Marie Curie IntraEuropean Fellowship, contract number MEIF-CT-2006-024476. E.R. and V.C. acknowledge financial support from the Junta de Andalucía and the Spanish Dirección General de Investigación (DGI) under project AYA2006-06375.

\section{References}

Baade, D. 1982, A\&A, 105, 65

Baade, D. 1989, A\&A, 222, 200

Balona, L. A. 1990, in Confrontation between stellar pulsation and evolution, ed.

C. Cacciari, \& G. Clementini, ASP Conf. Ser., 11, 245

Balona, L. A. 1995, MNRAS, 277, 1547

Balona, L. A., Cuypers, J., \& Marang, F. 1992, A\&ASS, 92, 533

Berthomieu, G., Gonczi, G., Graff, Ph., Provost, J., \& Rocca, A. 1978, A\&A 70, 597

Breger, M., Stich, J., Garrido, R., et al. 1993, A\&A, 271, 482

Carpino, M., Milani, A., \& Nobili, A. M. 1987, A\&A, 181, 182

Collins, G. W., Truax, R. J., \& Cranmer, S. R. 1991, ApJSS, 77, 541

Cuypers, J., Balona, L. A., \& Marang, F. 1989, A\&ASS, 81, 151

De Cat, P. 2002, in Radial and Nonradial Pulsations as Probes of Stellar Physics, ed. C. Aerts, T. R. Bedding \& J. Christensen-Dalsgaard, ASP Conf. Ser., 259,196
De Cat, P., \& Cuypers, J. 2003, in Interplay of Periodic, Cyclic and Stochastic Variability in Selected Areas of the H-R Diagram, ed. C. Sterken, ASP Conf. Ser., 292, 377

De Cat, P., Briquet, M., Aerts, C., et al. 2007, A\&A, 463, 243

Díaz-Cordovés, J., Claret, A., \& Giménez, A. 1995, A\&ASS, 110, 329

Dintrans, B., \& Rieutord, M. 2000, A\&A, 354, 86

Dupret, M.-A., De Ridder, J., De Cat, P., et al. 2003, A\&A, 398, 677

Dziembowski, W. A., \& Goode, Ph. 1992, ApJ, 394, 670

Dziembowski, W. A., Daszýnska-Daszkiewicz, J., \& Pamyatnykh, A. A. 2007, MNRAS, 374, 248

Fabregat, J., \& Reglero, V. 1990, MNRAS, 247, 407

Grenier, S., Baylac, M.-O., Rolland, L., et al. 1999, A\&AS, 137, 451

Gutiérrez-Soto, J., Fabregat, J., Suso, J., et al. 2006, in Astrophysics of Variable Stars, ed. C. Sterken \& C. Aerts, ASP Conf. Ser., 349, 249

Hanuschik, R. W., Hummel, W., Sutorius, E., Dietle, O., \& Thimm, G. 1996, A\&ASS, 116, 309

Harmanec, P. 1988, BaiCZ, 39, 329

Kupka, F., \& Bruntt H. 2001, in First COROT/MONS/MOST Ground Support Workshop, 39

Kuschnig, R., Weiss, W. W., Gruber, R., et al. 1997, A\&A, 328, 544

Montgomery, M. H., \& O’Donoghue, D. 1999, Delta Scuti Star Newsletter, 13, 28

Neiner, C., Hubert A.-M., \& Catala, C. 2005, ApJSS, 156, 237

Nielsen, R. F. 1983, Inst. Theor. Astrophys. Oslo Report, ed. O. Hauge, 59, 141

Perryman, M. A. C., Lindegren, L., Kovalevsky, J., et al. 1997, A\&A, 323, L49

Porter, J. M., \& Rivinius, Th. 2003, PASP, 115, 1153

Rivinius, Th., Baade, D., \& Štefl, S. 2003, A\&A, 411, 229

Saio, H. 1981, ApJ, 244, 299

Saio, H., Cameron, C., Kuschnig, R., et al. 2007, ApJ, 654, 544

Scargle, J.D. 1982, ApJ, 263, 835

Schrijvers, C., \& Telting, J. H. 1999, A\&A, 342, 453

Soufi, F., Goupil, M. J., \& Dziembowski, W. A. 1998, A\&A, 334, 911

Stankov, A., \& Handler G. 2005, ApJS, 158, 193

Townsend, R. 2003, MNRAS, 343, 125

Townsend, R. 2005, MNRAS, 364, 573

Valdes, F., Gupta, R., Rose, J. A., Singh, H. P., \& Bell, D. J. 2004, ApJS, 152, 251

Vaníček, P. 1971, Ap\&SS, 12, 10

Walker, G. A. H., Kuschnig, R., Matthews, J. M., et al. 2005, ApJ, 635, L77

Zamanov, R. K., Reig, P., Martí, J., et al. 2001, A\&A ,367, 884

Zorec, J. 2000, in The Be Phenomenon in Early-Type Stars, IAU Coll. 175, ed. M. A. Smith \& H. F. Henrichs, ASP Conf. Ser., 214, 51 
K. Uytterhoeven et al.: Multiperiodicity in the newly discovered mid-late Be star V2104 Cygni, Online Material p 1

\section{Online Material}


K. Uytterhoeven et al.: Multiperiodicity in the newly discovered mid-late Be star V2104 Cygni, Online Material p 2

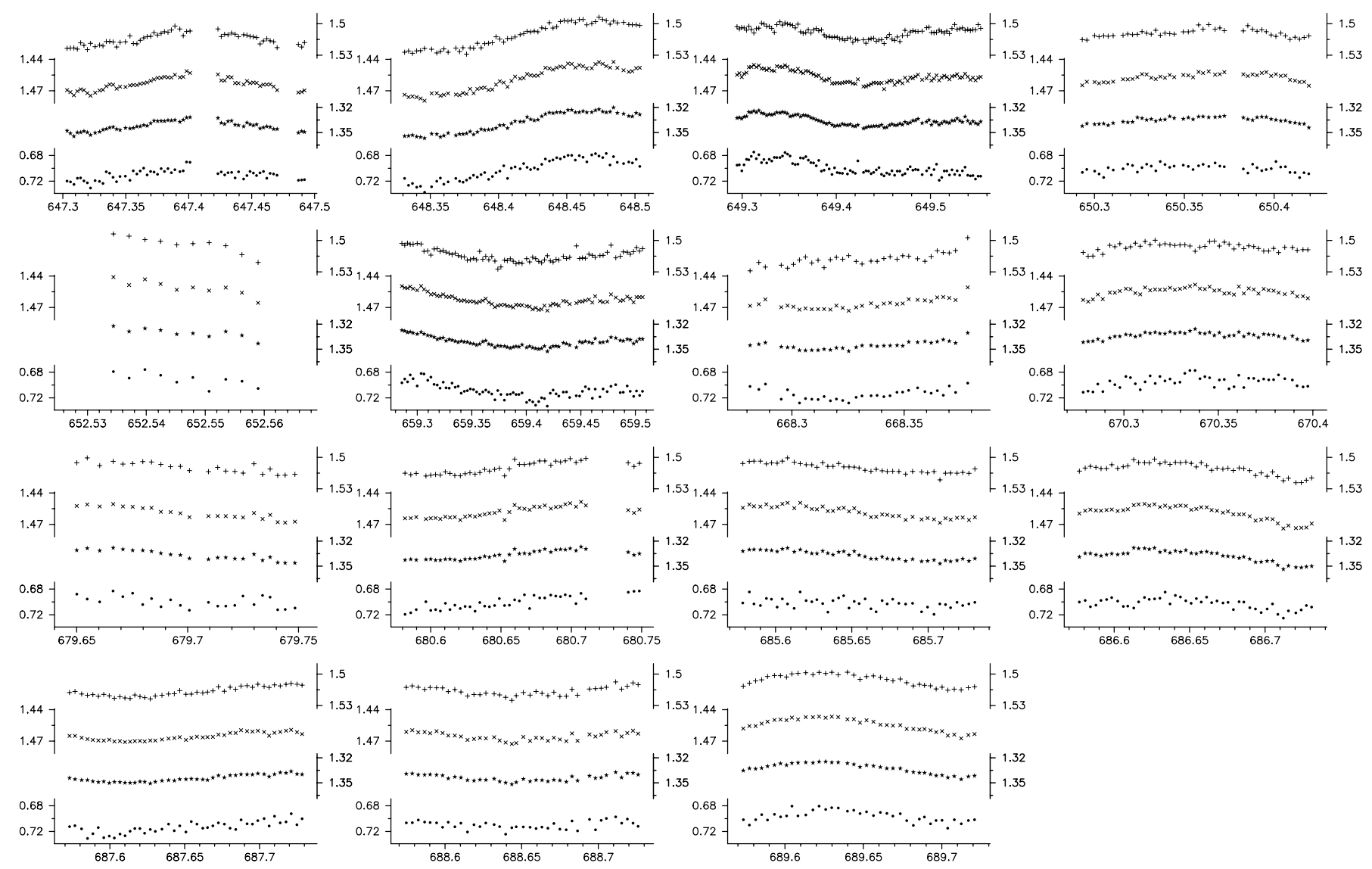

Fig. 1. Light curves of V2104 Cyg obtained at SNO and SPMO in October-November 2005. The $u$ (dots), $v$ (stars), $b$ (crosses) and $y$ (+) magnitude differences are plotted in time (HJD-2453000). Note the difference in scale between the $u$ and $v b y$ data: tickmarks in the $u$ filter and $v b y$ filters correspond to 0.04 mag and 0.03 mag respectively. 